Original article

\title{
The Comparability of Lipid-based and Body Mass Index-based Cardiovascular Disease Risk Scores: Using the Rwanda 2012- 2013 Non-communicable Diseases Risk Factors Survey Data
}

Jean Berchmans Niyibizi $1^{*}$, Okop Kufre Joseph ${ }^{2}$, Levitt Naomi², Stephen Rulisa ${ }^{3,8}$, Seleman Ntawuyirushintege ${ }^{1}$, David Tumusiime ${ }^{4}$, Alypio Nyandwi ${ }^{5}$, Evariste Ntaganda $^{6}$, Birhanu Ayele ${ }^{7}$, Charlotte Bavuma ${ }^{3,8}$

${ }^{1}$ Single Project Implementation Unit, University of Rwanda, Kicukiro, Kigali, Rwanda ${ }^{2}$ Chronic Diseases Initiative for Africa (CDIA), University of Cape Town, Rondebosch, Western Cape, South Africa

${ }^{3}$ School of Medicine and Pharmacy, College of Medicine and Health Sciences, University of Rwanda, Kigali, Rwanda

${ }^{4}$ School of Health Sciences, College of Medicine and Health Sciences, University of Rwanda, Kigali, Rwanda

${ }^{5}$ Republic of Rwanda Ministry of Health, Kigali, Rwanda

${ }^{6}$ Rwanda Biomedical Center, Kigali, Rwanda

${ }^{7}$ Division of Epidemiology and Biostatistics, Stellenbosch University, Stellenbosch, Western Cape, South Africa

8Kigali University Teaching Hospital, Kigali, Rwanda

*Corresponding author: Jean Berchmans Niyibizi. Single Project Implementation Unit, University of Rwanda, Kicukiro KK19 Av 101 P.O. Box 4285 Kigali, Rwanda. Email:

jeanberchmansniyibizi@yahoo.fr.

\section{Abstract}

\section{Background}

In Rwanda, cardiovascular diseases (CVDs) ranked second of the most common cause of death in 2016. CVD risk score tools have been recommended to identify people at high risk for management.

\section{Objective}

To assess the comparability of body mass index (BMI)-based and lipid-based CVD risk scores in Rwandan population.

\section{Methods}

Secondary analysis was conducted on 4185 study participants extracted from the dataset of Rwanda 2012-2013 non-communicable diseases risk factors 
survey. Individual CVD risk scores were calculated using both BMI-based and lipid-based algorithms, one at a time. Spearman rank's coefficient and Cohen's Kappa coefficient were used to compare the two tools.

\section{Results}

About $63.5 \%$ of participants were women. There was a significant positive correlation between BMI-based algorithm and lipid-based algorithm vis-à-vis a 10-year CVD risk prediction (Spearman rank correlation coefficients $>0.90$, $\mathrm{p}<0.001)$ considering either men, women or overall study participants. There was a moderate agreement between BMI-based and lipid-based algorithms visà-vis CVD risk characterization, kappa $=0.52 ; \mathrm{p}$-value $\mathrm{p}<0.001$ considering either overall study participants or men and kappa $=0.48 ; \mathrm{p}$-value $\mathrm{p}<0.001$ considering women.

\section{Conclusion}

The findings from this study suggest the use of BMI-based algorithm, a cost effective tool compared to lipid-based tool, can be alternatively used in resource-limited settings.

Rwanda J Med Health Sci 2021;4(1):166-184

Keywords: Cardiovascular diseases, algorithms, Rwanda

\section{Introduction}

Globally, cardiovascular disease (CVD) accounts for 17.9 million deaths annually, representing $31 \%$ of all global deaths and this makes them (CVDs) to be at the first position of deaths globally.[1] About $80 \%$ of all global CVD-related deaths occur in low- and middle-income countries (LMICs).[2] Sub-Saharan Africa (SSA) constituted 5.5\% (about 1 million) of CVD deaths in 2013.[3] Epidemiological data have indicated that SSA has a disproportionately high CVD mortality burden.[4,5]
In Rwanda, particularly, CVDs ranked third of the most common cause of death in 2012 accounting for $8 \%$ of deaths [6], and ranked second of the most common cause of death in 2016 accounting for 14 $\%$ of deaths.[7] These ranks of CVDs on proportional mortality in 2012 and 2016 confirm how CVDs should be among public health issues that need special attention in Rwanda.

In Rwanda, as it should be in SSA countries and other LMICs, urgent action is needed to lower the aforementioned CVD mortality burden. One of the strategies to 
achieve this goal (lowering CVD mortality burden), as it has been a case in developed countries, is to ensure early detection and treatment of individuals at risk. Lipid-based (L-B) techniques were initially developed, tested and applied to determine individual and absolute CVD risk prediction in developed countries.[8,9]These laboratory techniques were perceived to be costly in extent to which settings with limited resources could not apply them consistently and thus researches were conducted to develop BMIbased (BMI-B) algorithms, perceived cost-effective compared to L-B algorithms vis-à-vis determining CVD risk prediction and characterization.

The Framingham laboratory based equation (termed L-B equation in this paper) is one of the famous tools used to determine sex specific CVD risk prediction with use of eight CVD risk factors: Age, Total Cholesterol (T-C), high dose lipoprotein cholesterol (HDL_C), Systolic blood pressure (SBP), blood pressure (BP) treatment, smoking, and diabetes.[8] Afterwards, the Framingham non laboratory-based equation (termed BMI-based equation in this paper), which substitute body mass index (BMI) for T-C and HDL-C was developed and validated among African
Americans.[10] As the BMI-based equation does not require laboratory information, it is cost effective compared to lipid-based equation; reason why it has been recommended to be used, especially in resource-limited settings.[10,11] More studies were conducted to compare BMI-B equation to different $\mathrm{L}-\mathrm{B}$ equations and the results have indicated that there is positive correlation in CVD risk prediction and concordance in CVD risk characterization between lipid and BMI based equations.[11,12] However, all studies have recommended doing more studies in LMICs in order to evaluate the comparability of lipid and BMIbased algorithms.

On the best of our knowledge, neither approach utilizing lipidbased algorithm nor BMI-based algorithm was used in Rwanda to predict CVD risk in the community. BMI-based tool is the most accessible in resource-limited settings. In Rwanda, there is no intervention that has been initiated to screen individuals for high CVD risk using either BMI-based or lipidbased tool. This underpins a need to assess the comparability of both tools in predicting cardiovascular risk (CVR) to inform decision makers on which cost-effective tool could be used to identify individuals at high CVR for early treatment, 
prevention and reduction of CVD burden among Rwandan population. Thus, the objective of this study was to assess the comparability of L-B and BMI-B tools in predicting CVD risk score and characterizing CVD risk by conducting secondary analysis.

\section{Materials and methods}

\section{Type of study and data source}

We conducted a secondary analysis of World Health Organization Stepwise approach to NCD surveillance (WHO STEPs) subset of data collected during the NCDs risk factors population-based survey completed in 2013 in Rwanda. The survey was a cross-sectional survey and the participants were between 15 and 65 years old. The general research methodology, ethical considerations and results for the first NCD risk factors survey are described elsewhere.[13]

Access to STEPs dataset was authorized and provided by Rwanda Biomedical Centre (RBC), a policy implementing agency of Rwanda Ministry of Health. The primary survey included 7240 participants. During the data cleaning, we omitted study participants with missing data and non-response on any key variables to be used in calculation of individual total CVD risk score for both lipid and BMI based equations, and 6613 study participants remained. From the 6613 participants, we excluded those below 30 years old as the lipid-based CVD risk scoring tool (Framingham heart Study) was established based on a population of 30 years old and above. A total of 4185 was included in the final dataset to determine an individual and absolute total CVD risk score using both lipid-based and BMIbased equations.

Individual-levels absolute CVD risk scores were calculated using both tools taking into consideration selfreported data (sex, age, diabetes, high blood pressure treatment, and current smoking status), anthropometric measurement data (height, weight, calculated body mass index and systolic blood pressure), and laboratory data (total cholesterol; T-c and High Density Lipoprotein-C: HDL-c). The lipidbased (L-B) algorithm tool provides 10-year cardiovascular risk score basing on age, T-c, HDL-c, systolic blood pressure and high blood pressure treatment. The BMI-based algorithm provides the 10-year global cardiovascular risk score using all variables in lipid-based algorithm except T-c and HDL-c which are replaced by the BMI. It is to note that in primary data we had three SBP readings, and in this 
secondary study the average between the second and third SBP readings was calculated and considered as the real SBP for included participant. The variable of diabetes, in present study, encompasses both newly diagnosed and reported cases. With reference to the WHO standards, [14] the newly diagnosed diabetes was confirmed when fasting blood glucose is $\geq 7 \mathrm{mmol} / 1(126 \mathrm{mg} / \mathrm{dl})$.

To calculate the individual global CVD risk scores, we used the formulae employed by D'Agostino et al: [15] CVD risk score $=1$ -

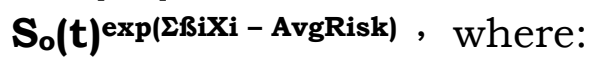

- $S o(t)$ is the baseline survival at follow-up time $t$ (here $t=10$ years) as determined in the Framingham Heart Study,

- $\mathrm{Bi}$ is the estimated regression coefficient as determined in the Framingham Heart Study,

- $\mathrm{Xi}$ is log-transformed value of the $i^{\text {th }}$ risk factor as provided in dataset of the first NCDs risk factors survey,

- AvgRisk is an average risk as determined in the initial Framingham Heart Study.
Because of time and financial constraints to conduct a 10-years cohort study we used So(t), $\beta i$ and AvgRisk as determined in the Framingham heart study and their respective values are provided per gender (sex) and per type of algorithm in the Table 1. However, we acknowledge that there exists a difference between population from Framingham heart study and Rwandan population and thus the results should be interpreted with caution. 
Table 1. So (10), AvgRisk and $\beta i$ per type of gender (sex) and per algorithm (separately)

\begin{tabular}{|c|c|c|c|}
\hline Algorithm & Variable & Women & Men \\
\hline \multirow{9}{*}{ 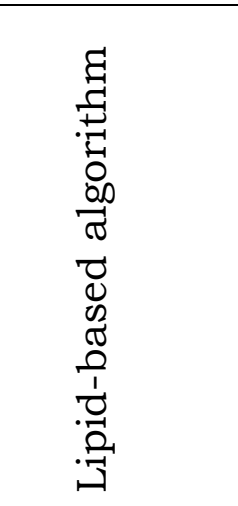 } & So (10) & 0.95012 & 0.88936 \\
\hline & AvgRisk & 26.1931 & 23.9802 \\
\hline & $\beta$ for age & 2.32888 & 3.06117 \\
\hline & $\beta$ for total cholesterol & 1.20904 & 1.12370 \\
\hline & $\beta$ for HDL cholesterol & -0.70833 & -0.93263 \\
\hline & $\beta$ for SBP if not treated & 2.76157 & 1.93303 \\
\hline & $\beta$ for SBP if treated & 2.8226 & 1.99881 \\
\hline & $\beta$ for Smoking & 0.52873 & 0.65451 \\
\hline & $\beta$ for Diabetes & 0.69154 & 0.57367 \\
\hline \multirow{8}{*}{ 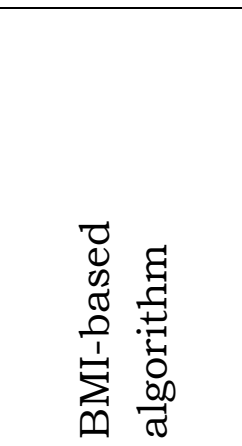 } & So (10) & 0.94833 & 0.88431 \\
\hline & AvgRisk & 26.0145 & 23.9388 \\
\hline & $\beta$ for age & 2.72107 & 3.11296 \\
\hline & $\beta$ for BMI & 0.51125 & 0.79277 \\
\hline & $\beta$ for SBP if not treated & 2.81291 & 1.85508 \\
\hline & $\beta$ for SBP if treated & 2.88267 & 1.92672 \\
\hline & $\beta$ for Smoking & 0.61868 & 0.70953 \\
\hline & $\beta$ for Diabetes & 0.77763 & 0.53160 \\
\hline
\end{tabular}

So (10): Baseline survival at follow-up time $\mathrm{t}(\mathrm{t}=10$ years), AvgRisk: Average risk, ßi: Regression coefficient, HDL: High Density Lipoprotein, SBP: Systolic Blood Pressure, BMI: Body mass index

\section{Statistical analysis}

Box plot and histogram were used to assess the distribution of continuous variables, and descriptive statistics were used to describe the included participants. The mean ( \pm standard deviation) was used to present normally distributed continuous variables, otherwise the median $\left(25^{\text {th }}-75^{\text {th }}\right.$ percentiles) was used. Categorical variables were presented in both absolute frequencies and percentages. To compare men and women vis-à-vis CVD risk factors (variables) considered to calculate CVD risk scores for both algorithms (L-B and BMI-B), Pearson's Chi-squared $\left(\mathrm{x}^{2}\right)$ and Student's t-tests were used for categorical and continuous variables, respectively. Wilcoxon rank-sum test was applied for continuous variables with asymmetric distribution. Homoscedasticity was assessed using Levene's test and Fmax test before conducting Student's t-test 
for continuous variables with symmetric distribution.

Spearman rank's correlation coefficient was used to assess the correlation between the BMI-based algorithm and lipid-based algorithm in determining a 10-year CVD risk score. Scatter plots were used to present the correlation between the two types of individual CVD risk scores. As indicated in one of the previous comparative studies,[16] CVD risk was categorized as: $<10 \%$ low risk, $[10 \%-20 \%[$ moderate risk and $\geq 20 \%$ high risk. The percent of study participants equivalently characterized as 'high', 'moderate' or 'low' risk was determined in the present study for the lipid-based and BMI-based equations of the two CVD risk scores. Statistical testing procedures for Cohen's Kappa were conducted to test the level of agreement between these two algorithms in ranking study participants in three categories of CVD risk. All statistical analyses were performed using Stata 13 (Stata Corporation, College Station, Texas, USA); and significance testing was performed at $5 \%$.

\section{Ethical considerations}

The Institutional Review Board at Rwanda Ministry of Health, known as Rwanda National Ethics
Committee (RNEC), evaluated the protocol of this study and approved it (No.256/RNEC/2019).

\section{Results}

\section{Input cardiovascular risk variables}

A total of 4185 participants were included for the analysis, among which 2657 (63.5\%) were women. The median age (years), total cholesterol $(\mathrm{mg} / \mathrm{dl})$ and the mean BMI $\left(\mathrm{Kg} / \mathrm{m}^{2}\right)$ among women are significantly higher compared to those of men (see Table 2). The mean SBP $(\mathrm{mmHg})$, the prevalence of reported smoking for men is significantly higher compared to that of women (see Table 2). Median HDL cholesterol is significantly lower in men than in women. Reported hypertension treatment rate was similarly low in men and women, and the prevalence of diabetes is statistically the same between men and women (see Table 2). 
Table 2. Input CVR variables and gender

\begin{tabular}{lllll}
\hline Characteristic & Total (N= 4185) & Men (n=1528) & Women (n=2657) & P-value \\
\hline $\begin{array}{l}\text { Age, median } \\
\text { (P25-P75) }\end{array}$ & $41(34-50)$ & $40(34-50)$ & $41(34-51)$ & $0.036^{*}$ \\
$\begin{array}{l}\text { Total } \\
\text { cholesterol, } \\
\text { median (P25- }\end{array}$ & $120.3(100.2-143.1)$ & $115.2(100.2-136.7)$ & $123.4(104.0-146.9)$ & $<0.001^{*}$ \\
$\begin{array}{l}\text { P75), mg/d1 } \\
\text { HDL, median }\end{array}$ & $39.8(30.5-52.2)$ & $37.5(29.0-50.3)$ & $41.0(31.7-53.0)$ & $<0.001^{*}$ \\
$\begin{array}{l}\text { (P25-P75), } \\
\text { mg/dl }\end{array}$ & & & & $0.010^{*}$ \\
$\begin{array}{l}\text { SBP (Mean, } \\
\text { SD), mmHg } \\
\text { BP treatment, } \\
\text { n (\%) }\end{array}$ & $29(0.7)$ & $124.98(15.51)$ & $123.53(18.55)$ & 0.076 \\
$\begin{array}{l}\text { Smoking, n } \\
\text { (\%) } \\
\text { Newly } \\
\text { diagnosed and } \\
\text { reported } \\
\text { diabetes, n (\%) } \\
\text { BMI (Mean, } \\
\text { SD) }\end{array}$ & $826(19.7)$ & $6(0.4)$ & $23(0.9)$ & $<0.001^{*}$ \\
\hline
\end{tabular}

HDL: High Density Lipoprotein, SBP: Systolic Blood Pressure, BP: Blood pressure, BMI: Body Mass Index, *Statistical significance between men and women $(\mathrm{P}$-value $\leq 0.05)$

1.Gender and 10 year CVR score prediction using BMI and Lipid based tools

Both tools found that men are at high risk to develop cardiovascular event at 10 years. The median $\left(25^{\text {th }_{-}}\right.$ $75^{\text {th }}$ percentiles) 10 -year absolute CVD risk scores predicted by both
BMI-based and lipid based tools among men are significantly greater compared to women; $5.09 \%(2.8$ $10.33)$ vs $2.48 \%(1.36-5.24)$ with $\mathrm{p}$ value $<0.001$ and $3.61 \%$ (1.98$7.26)$ vs $1.86 \%$ (1.07-3.75) with $\mathrm{p}$ value < 0.001, respectively (See Table 3). 
Table 3. The 10-year CVD risk prediction by each algorithm

\begin{tabular}{lllll}
\hline Algorithms & $\begin{array}{l}\text { Total } \\
(\mathbf{N = 4 , 1 8 5 )}\end{array}$ & $\begin{array}{l}\text { Men } \\
(\mathbf{n = 1 , 5 2 8 )}\end{array}$ & $\begin{array}{l}\text { Women } \\
(\mathbf{n = 2 , 6 5 7 )}\end{array}$ & $\begin{array}{l}\text { p- } \\
\text { value* }\end{array}$ \\
\hline $\begin{array}{l}\text { A 10-year CVD risk score } \\
\text { prediction by BMI-based } \\
\text { tool, median (P25-P75) }\end{array}$ & $3.27 \%$ & $5.09 \%$ & $2.48 \%$ & $<0.001$ \\
& $(1.72-6.93)$ & $(2.8-10.33)$ & $(1.36-5.24)$ & \\
& & & & \\
$\begin{array}{l}\text { A 10-year CVD risk score } \\
\text { prediction by lipid-based } \\
\text { tool, median (P25-P75) }\end{array}$ & $2.37 \%$ & $3.61 \%$ & $1.86 \%$ & $<0.001$ \\
& $(1.28-4.95)$ & $(1.98-7.26)$ & $(1.07-3.75)$ & \\
\hline
\end{tabular}

\footnotetext{
*Indicates statistical significance between men and women, BMI: Body Mass Index BMI-based tools, BMI-based Although, the proportions of study algorithm seems to detect more participants classified in moderate and high 10-years CVR (cardiovascular risk) are people at moderate and high risk than lipid-based algorithm significantly higher among men compared to women as per the prediction by both lipid-based and
} 
Table 4. Characterization of Study Participants into three Categories of CVD Risk

\begin{tabular}{|c|c|c|c|c|c|}
\hline Algorithms & $\begin{array}{l}\text { A 10-year } \\
\text { CVD Risk }\end{array}$ & $\begin{array}{l}\text { Total } \\
(\mathrm{N}=4,185)\end{array}$ & $\begin{array}{l}\text { Men } \\
(n=1,528)\end{array}$ & $\begin{array}{l}\text { Women } \\
(n=2,657)\end{array}$ & p-value* \\
\hline \multirow{3}{*}{$\begin{array}{l}\text { Ranking by } \\
\text { BMI-based } \\
\text { Algorithm, } n \\
\text { (\%) }\end{array}$} & Low risk ${ }^{1}$ & $3,537(84.5)$ & $1,132(74.1)$ & $2,405(90.5)$ & $<0.001$ \\
\hline & Moderate risk ${ }^{2}$ & $465(11.1)$ & $263(17.2)$ & 202 (7.6) & \\
\hline & High risk ${ }^{3}$ & $183(4.4)$ & $133(8.7)$ & 50 (1.9) & \\
\hline $\begin{array}{l}\text { Ranking by } \\
\text { Lipid-based }\end{array}$ & Low risk ${ }^{1}$ & $3,812(91.1)$ & 1,275 (83.5) & $2,537(95.5)$ & $<0.001$ \\
\hline \multirow{2}{*}{$\begin{array}{l}\text { Algorithm, n } \\
(\%)\end{array}$} & Moderate risk ${ }^{2}$ & 287 (6.9) & $190(12.4)$ & $97(3.6)$ & \\
\hline & High risk ${ }^{3}$ & $86(2.0)$ & $63(4.1)$ & $23(0.9)$ & \\
\hline
\end{tabular}

1.Agreement between BMI and Lipid based CVR scoring tools to predict a 10-year CVR score

There is a strong positive correlation between Lipid-based and BMI-basedalgorithms in predicting a 10-year CVD risk score in overall participants, women and men (see

Figure of scatterplots) with spearman rank correlation coefficients (SRCC) of $0.9380,0.9086$ and 0.9399, respectively (all p-values for the SRCC were $<0.001)$. 
Figure of scatterplots: Scatter plots indicating the performance of BMIbased tool vs Lipid-based tools
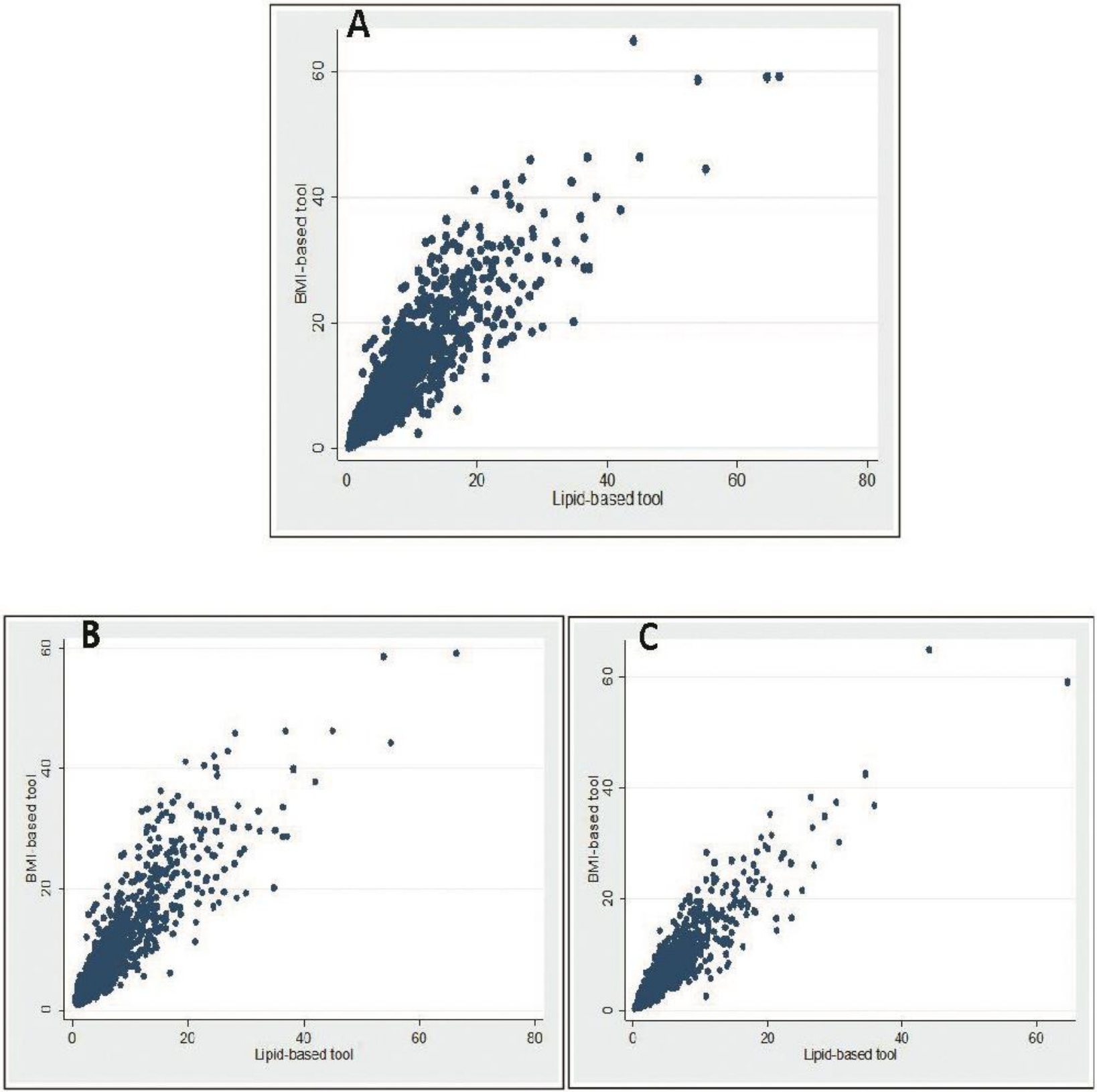

A: Scatter plot for overall study participants; B: Scatter plot for men; C: Scatter plot for women, BMI: Body mass index

The observed agreement between the two algorithms was at $89.3 \%$ with kappa statistic of 0.52 (P-value
$<0.001)$. When we computed kappa statistic for men only and women, one at a time, we found that the observed agreement was at $82.9 \%$ 
with kappa statistic of 0.52 (pvalue $<0.001)$ and $93.1 \%$ with kappa statistic of 0.48 (p-value $<0.001$ ), respectively. Thus, the agreement found between L-B algorithm and BMI-B algorithm in determining CVD risk score for three cases is moderate, as three kappa statistics are in the range of $0.41-0.60,[17]$ and is statistically significant (Pvalue $<0.001)$. The reason for the discrepancy between the unadjusted level of agreement and kappa found in this study is that high CVD risk is a rare observation.

\section{Discussion}

Cardiovascular diseases are among the first causes of morbidity and mortality globally but mostly in LMICs. Early detection and treatment of individuals at high CVR may alleviate the burden caused by CVD. Finding a most accessible and cost-effective tool to predict CVR is an essential solution in LMIC. In this study, we assessed the similarity of lipid-based and BMIbased models in predicting the total risk to develop cardiovascular disease.

Gender distribution of traditional cardiovascular risks varies globally depending on studied population.[18] In the present study, we found that men were at high cardiovascular risk than women using both lipid-based and BMI- based tool (Table 3). This indicates that both the two tools have tendency of predicting CVD risk score in the same direction. This finding corroborates with other existing evidence.[19] This may be helpful in prevention and health education strategies.

The median $\left(25^{\text {th }}-75^{\text {th }}\right.$ percentiles) estimated 10-year CVD risk score to the overall participants in this study, $3.27 \%$ (1.72-6.93) for BMIbased algorithm and $2.37 \%(1.28-$ 4.95) for lipid-based algorithm are quite lower than those estimated in the study conducted among Ghanaian migrant and home populations. In the latter study, estimated median $\left(25^{\text {th }}-75^{\text {th }}\right.$ percentiles) was $9.5 \%$ (5.4-15.7) for Framingham non-laboratory (BMIbased) algorithm and 7.3\% (3.913.2) for Framingham laboratory (lipid-based) algorithm.[20] This difference may be due to the difference in CVD risk factors profile between two study populations; CVD risk factors profile of Ghanaian population [20] indicates that unhealthy lifestyles and metabolic risk factors for CVDs (not reported here) are more prevalent among Ghanaian population than among Rwandan population (Table 2).

Even though laboratory-based CVD risk assessment is taken as a gold 
standard[11], we found that the proportions of study participants in high and moderate CVD risk categories are higher for nonlaboratory based algorithm compared to laboratory based algorithm, for both men and women. These findings corroborate with the study conducted in Ghana, an African country, where it was found that the non-laboratory equation ranked almost 1.5 times more people at high absolute 10-year CVD risk than did the laboratory equation.[20] These findings are consistent with the study of Pandya and his colleagues who found that the non-laboratory-based risk score similarly characterized high and low risk men and women compared to the Framingham function.[11] This finding may be taken as an advantage of using the BMI-based tool in the community with a referral guideline to health facilities for confirmation and treatment. It would be a concern if BMI-based tool has underestimated the CVR prediction.

When we assessed the correlation between lipid-based algorithm and BMI-based algorithm to predict a 10-year CVD risk for men, women and overall study participants, we found that there is a positive correlation for all cases (the spearman rank correlation coefficients $>0.90)$ and is statistically significant $(\mathrm{p}<0.001)$. This level of correlation is considered a very high correlation as in all cases (overall study participants, men only and women only) spearman rank correlation is greater than 0.90.[21] The results from the correlation assessment are not far from the results found in other comparative studies conducted to compare laboratorybased risk score to non-laboratorybased risk score and found that the Spearman rank correlation coefficients is greater than 0.90.[11,12,22] With these findings, it may be assumed that BMI-based and lipid-based algorithm are comparable in CVD risk score prediction, irrespective of the settings. However, more studies are needed in the settings where such a study has not yet been conducted on the comparability of the two tools.

Furthermore, we computed kappa statistics to assess an agreement between BMI-based algorithm and lipid-based algorithm, and found that there is a moderate agreement between two tools as kappa statistic equals 0.52 (P-value <0.001), 0.52 (p-value $<0.001)$ and $0.48 \quad(p-$ value $<0.001)$ considering overall study participants, men only and women only, respectively. However, 
the kappa statistics, found in this study, is lower than the one found in the study [20] when Framingham laboratory and non-laboratory based tools were compared, kappa statistics was 0.63 . This difference may have resulted either from study design of current study (crosssectional) which is different from the study design of Framingham heart study (cohort study) or difference between study populations in various aspects of life. In availability of (financial) resources, a cohort study used in Framingham heart study would be ideal to exactly quantify and confirm the reason why kappa statistics found in this study are lower than the ones found in the study conducted by Daniel et al. [20]

\section{Study limitation}

Even though the dataset from which the study participants were drawn was designed in such a way as to be representative of the Rwandan population, the sample of this study might be not quite representative of the Rwandan population due to the eligibility criteria. The sample excluded study participants below 30 years of age, participants with missing data and non-response on any key variables required to calculate the individual total CVD risk score for both lipid and BMI based equations. Despite this limitation of the incompleteness / non response, further investigation indicated that incompleteness is not participants' gender or age dependent (results not shown here), thus we assume that the incompleteness is missing completely at random (MCAR). This implies that the results of this study are unbiased. In addition, diabetes diagnosis was based on fasting blood glucose or history of diabetes in a setting where the screening of diabetes is not routinely done. However, the criteria for diabetes status was the same for both tools. Waist circumference (WC) is not considered in both tools while high WC reflects visceral fat accumulation which is prone to cause cardiovascular events in LMICs.[23]

In this study, even though CVD risk scores were not calculated from actual or updated data because of time and financial constraints to conduct a 10-year cohort study, the present findings can be used as a baseline of further studies assessing comparability of both BMI-based and lipid-based algorithms in predicting individual CVD risk score. We also acknowledge the difference between population from Framingham heart study and Rwandan population, and thus we recommend, in availability of financial resources and time, to 
conduct a 10-year or 5-year cohort study to determine an accurate CVD risk core.

\section{Conclusion}

BMI-based and lipid-based algorithms agree satisfactorily and highly correlate in predicting the total 10-years cardiovascular risk in Rwanda. As stated from other works, BMI-based algorithm should be used in situations with limited resources where laboratory facilities are inaccessible. In Rwandan specific context, we recommend its use at the community level with referral guideline; however, there is need to evaluate how easy it is to be used by community health workers. There is gender disparity in cardiovascular risk characterization; men being at higher risk than women. Further studies to understand this gender disparities and determinants of high cardiovascular risk are needed to contextualize interventions.

\section{Authors' contributions}

CBM, JBN, KJO, NL and EN contributed to the conception or design of the work (secondary study). CBM, EN, SR and SN contributed in the acquisition of the data. BTA contributed to statistical analysis, JBN performed statistical analysis and is accountable for accuracy and data integrity. JBN and CBM drafted the manuscript. CBM, JBN, SR, DT, AN, EN, SN, SR, BTA, KJO and NL critically revised and commented on the manuscript. All authors read and approved the final manuscript.

\section{Competing Interests}

The authors declare that they have no competing interest.

\section{Funding}

This work was funded by German Federal Ministry of Education and Research (BMBF) as part of the Research Networks for Health Innovations in sub-Saharan Africa Funding Initiative through Collaboration for Evidence-based Healthcare and Public Health in Africa (CEBHA+) project (Number: 66.3010.7-002.09). The sponsor did not take part in any phase of the study; neither study design, data collection, data analysis, interpretation of data, writing the report nor in the decision to submit the article for publication

This article is published open access under the Creative Commons Attribution-NonCommercial NoDerivatives (CC BYNC-ND4.0). People can copy and redistribute the article only for noncommercial purposes and as long as they give appropriate credit to the authors. They cannot distribute any modified material obtained by remixing, transforming or building upon this article. See https: / / creativecommons.org/licenses/by-nc$\mathrm{nd} / 4.0$ / 


\section{References}

1. WHO. Cardiovascular diseases ( CVDs ), 2017. https://www.who.int/en/new s-room/factsheets/detail/cardiovasculardiseases-(cvds) [Accessed on May 8, 2020];

2. Bowry ADK, Lewey J, Dugani SB, Choudhry NK. The Burden of Cardiovascular Disease in Low- and MiddleIncome Countries: Epidemiology and Management. Can J Cardiol. 2015;31:1151-1159.

doi:10.1016/j.cjca.2015.06.02 8.

3. Mensah GA, Roth GA, Sampson UK et al. Mortality from cardiovascular diseases in sub-Saharan Africa, 19902013: a systematic analysis of data from the Global Burden of Disease Study 2013. Cardiovasc J Afr. 2015;26:S6S10.doi:10.5830/CVJA-201536.

4. Keates AK, Mocumbi AO, Ntsekhe M, Sliwa K SS. Cardiovascular disease in Africa: epidemiological profile and challenges. Nat Rev
Cardiol. $\quad 2017 ; 14: 273-293$. doi:10.1038/nrcardio.2017.19

5. D'Agostino RB, Vasan RS, Pencina MJ, Wolf PA, Cobain M, Massaro JM, et al. General cardiovascular risk profile for use in primary care: The Framingham heart study. Circulation. 2008;117:743-53.

6. Rwanda Ministry of Health. Non Communicable Diseases Policy, 2015. http://moh.gov.rw/fileadmin/ templates/policies/NCDs_Poli cy.2015.pdf [Accessed on December 16, 2019];

7. WHO. Noncommunicable Diseases (NCD) Country Profiles, 2018. https://www.who.int/nmh/co untries/rwa_en.pdf?ua $=1$ [Accessed on May 8, 2020];

8. Yusuf S, Rangarajan S, Teo K et al. Cardiovascular risk and events in 17 low-, middle-, and high-income countries. $N$ Engl J Med. 2014;371:818827.

doi:10.1056/NEJMoa1311890

9. Rücker V, Keil U, Fitzgerald 
AP et al. Predicting 10-Year Risk of Fatal Cardiovascular Disease in Germany: An Update Based on the SCOREDeutschland Risk Charts. PLoS One. 2016;11:e0162188. Published $2016 \quad$ Sep 9.doi:10.1371/journal.

10. Kariuki JK, Gona P, Leveille SG, Stuart-Shor EM, Hayman LL CJ. Cost-effectiveness of the non-laboratory based Framingham algorithm in primary prevention of cardiovascular disease: A simulated analysis of a cohort of African American adults. Prev Med. 2018;111:415-422. doi:10.1016/j.ypmed.2017.12. 001.

11. 11. Pandya A, Weinstein MC GT. A Comparative Assessment of NonLaboratory-Based versus Commonly Used LaboratoryBased Cardiovascular Disease Risk Scores in the NHANES III Population. PLoS One. 2011;6:e20416.

doi:10.1371/journal.pone.002 0416.

12. Gaziano TA, Pandya A, Steyn $\mathrm{K}$ et al. Comparative assessment of absolute cardiovascular disease risk characterization from nonlaboratory-based risk assessment in South African populations. BMC Med. 2013;11:170. Published 2013 Jul 24. doi:10.1186/17417015 .

13. Ministry of Health. Rwanda Non-communicable Diseases Risk Factors Report, 2015. https://www.who.int/ncds/s urveillance/steps/Rwanda_20 12_STEPS_Report.pdf

[Accessed on December 12,2020];

14. WHO. Definition and diagnosis of diabetes mellitus and intermediate hyperglycemia: Report of a WHO/IDF consultation, 2016. https: / / www.who.int/diabete s/publications/Definition $\% 20$ and\%20diagnosis\%20of\%20di abetes_new.pdf [Accessed on January 8, 2020];

15. D'Agostino RB Sr, Vasan RS, Pencina MJ et al. General cardiovascular risk profile for use in primary care: the Framingham Heart Study. Circulation. 2008;117:743753. 
doi:10.1161/CIRCULATIONA

HA. 107.699579.

16. Kariuki JK, Stuart-Shor EM, Leveille SG, Gona P, Cromwell $\mathrm{J}$ HL. Validation of the nonlaboratory-based

Framingham cardiovascular disease risk assessment algorithm in the Atherosclerosis Risk in Communities dataset. $J$ Cardiovasc Med. 2017;18:936945.doi:10.2459/JCM.000000 0000000583.

17. Kottner J, Audigé L, Brorson S, Donner A, Gajewski BJ, Hróbjartsson A, et al. Guidelines for reporting reliability and agreement studies (GRRAS) were proposed. $J$ Clin Epidemiol. 2011;64:96-106.

18. Mosca L, Barrett-Connor E WN. Sex/gender differences in cardiovascular disease prevention: what a difference a decade makes. Circulation. 2012;124:2145-2154. doi:10.1161/CIRCULATIONA HA. 110.968792.

19. Kanchi R, Perlman SE, Chernov $\mathrm{C}$ et al. Gender and
Race Disparities in Cardiovascular Disease Risk Factors among New York City Adults: New York City Health and Nutrition Examination Survey (NYC HANES) 20132014. J Urban Heal. Journal of Urban Health; 2018;95:801812. doi: $10.1007 / \mathrm{s} 11524-$ 018-0287-x.

20. Boateng D, Agyemang C, Beune $\mathrm{E}$ et al. Cardiovascular disease risk prediction in subSaharan African populations Comparative analysis of risk algorithms in the RODAM study. Int $J$ Cardiol. The Authors; 2018;254:310-315. doi:10.1016/j.ijcard.2017.11. 082 .

21. 21. Mukaka MM. Statistics corner: A guide to appropriate use of correlation coefficient in medical research. Malawi Med J. 2012;24:69-71.

22. Peer N, Lombard C, Steyn K et al. Comparability of total cardiovascular disease risk estimates using laboratory and non-laboratory based assessments in urbandwelling South Africans: the CRIBSA study. S Afr Med J. 
2014;104:691-696.Published

$2014 \quad$ Aug 13.

doi: $10.7196 /$ samj. 81 .

23. Ross R, Neeland IJ, Yamashita $\mathrm{S}$ et al. Waist circumference as a vital sign in clinical practice: a Consensus Statement from the IAS and ICCR Working Group on Visceral Obesity. Nat Rev Endocrinol. Springer US; 2020;16:177-189. doi: $10.1038 / \mathrm{s} 41574-019-$ 0310-7. 